\title{
The effect of stress and anxiety associated with maternal prenatal diagnosis on feto-maternal attachment
}

\author{
Sara J Allison ${ }^{1 *}$, Julie Stafford ${ }^{2}$ and Dilly OC Anumba $^{3}$
}

\begin{abstract}
Background: A couple's decision to undergo an invasive test based on a screening test result is a process associated with anxiety. The aim of this study was to determine whether anxiety and prenatal attachment were affected by undergoing an invasive test compared to women in early pregnancy and after a reassuring anomaly scan.

Methods: 200 women were recruited at booking, 14 women and 20 partners after an invasive test and 81 women following an anomaly scan. A questionnaire was completed using the Beck Anxiety Inventory and Maternal or Paternal Antenatal Attachment Scales.

Results: Women who have had an invasive test have higher levels of anxiety compared to women at booking ( $P$ $<0.01)$ and after an anomaly scan $(P=0.002)$. Anxiety declines from booking to the time of an anomaly scan $(P=$ 0.025), whilst attachment increases $(P<0.001)$. There is a positive correlation between anxiety and attachment in women who have had an invasive test $(r=0.479)$. Partners of women undergoing an invasive test experience lower levels of anxiety $(P<0.05)$.

Conclusions: Women undergoing prenatal diagnostic procedures experience more psychological distress, which may be currently underestimated. Establishment of interdisciplinary treatment settings where access to psychological support is facilitated may be beneficial.
\end{abstract}

\section{Background}

Prenatal screening for chromosomal abnormalities such as Down's syndrome is offered to all pregnant women in the United Kingdom as part of routine antenatal care. This initial screening process provides the mother-to-be and her partner with an estimation of the risk that their child may have an abnormality. The decision to undergo further more invasive diagnostic tests based on this probability then rests with the couple. The process of making this decision is associated with increased anxiety and stress for the pregnant woman and her partner.

The levels of stress and anxiety may be influenced by the information given, the counselling and psychological support provided, the results of the prenatal tests, and the outcome of the pregnancy. Stress and anxiety may

\footnotetext{
* Correspondence: sarajaneallison@gmail.com

${ }^{1}$ Academic Unit of Reproductive and Developmental Medicine, University of Sheffield, 4th Floor, Jessop Wing, Tree Root Walk, Sheffield, S10 2SF, UK

Full list of author information is available at the end of the article
}

also affect maternal feelings of attachment towards the fetus, and it is plausible that appropriate care and support may positively influence feto-maternal attachment by minimising stress.

\section{Anxiety in Pregnancy}

Anxiety is defined as the psychological consequence of exposure to a real or imagined stress [1]. The decision to undergo a prenatal invasive test is undoubtedly associated with anxiety. There is much debate surrounding prenatal invasive testing as a means of reassurance for women identified as being at risk of carrying a fetus affected by a chromosomal or structural abnormality. While undergoing prenatal screening for genetic abnormalities may protect women from high levels of anxiety $[2,3]$, it may also encourage women to focus on what may be wrong with the child, increasing levels of anxiety [4].

\section{C) Biomed Central}


Previous research has clearly shown a relationship between undergoing an invasive test and increased anxiety levels $[2,5,6]$ although receiving a normal result may in fact reduce the anxiety experienced later in the pregnancy [3].

Studies of prenatal anxiety and stress are important because there is increasing evidence that prenatal anxiety and stress may have long-term sequelae for both the pregnant woman and her fetus [7-9]. The mechanism by which these adverse effects occur is poorly understood but animal studies have shown that chronic stress may downregulate the fetal cortisol barrier enzyme response resulting in enhanced exposure of the fetus to maternal cortisol levels [10]. Abnormally high placental corticotropin releasing hormone (CRH) levels can cause vasodilatation resulting in reduced oxygen and nutrient delivery to the fetus [11]. Prolonged suboptimal conditions may lead to a state of 'thrifty' metabolism [12] predisposing to type II diabetes and obesity in later life [13]. If this physiological fetal programming occurs at a stage when the fetus is particularly sensitive to stressors there may be long-lasting impact upon memory, learning, affect and even frontal lobe executive function [9]. A study by Uno et al found that animals treated with synthetic glucocorticoids prenatally were shown to have a $30 \%$ reduction in hippocampal size [14].

Furthermore, stimulation of the autonomic nervous system (ANS) by anxiety or stress may lead to increased release of catecholamines such as noradrenaline, increasing uterine artery resistance and arterial pressure, causing a decrease in uterine blood flow and thus oxygen delivery to the fetus. One study found that high noradrenaline levels in pregnancy were negatively correlated to fetal head and abdominal circumferences [15].

\section{Prenatal Attachment}

Any doubts raised concerning the health of the fetus that may lead to increased levels of stress and anxiety, such as a positive screening result or prenatal invasive test, may also interrupt the normal development of the psychological bond between the other and her fetus, which could lead to abuse, neglect and the ill-health of the child.

Feto-maternal attachment was described by Muller as 'the unique and affectionate antenatal relationship that develops between a mother and fetus' and it is characterised by the behaviours, attitudes, thoughts and feelings that demonstrate care and commitment to the fetus $[16,17]$. There is little previous research into prenatal attachment in women undergoing screening or an invasive diagnostic test in pregnancy. One study, using the Pregnancy Involvement List (PIL) to measure attachment, found that offering screening to women temporarily increases attachment [18] but another showed that undergoing a prenatal invasive test may decrease prenatal attachment, assessed using the Prenatal Attachment Interview (PAI) [19].

Two small studies have suggested that men have lower anxiety levels than their partners at the time of a prenatal invasive test $[20,21]$ but did not report on paternal attachment.

\section{Purpose of the Study}

This study aims to compare the relationship between anxiety and attachment in women and their partners undergoing an invasive test to women at an early gestation (who have not yet had any screening) and women at about 20 weeks gestation whose fetal morphology scan has shown no apparent abnormalities.

\section{Methods}

Pregnant women and their partners attending the Jessop Wing, Royal Hallamshire Hospital, Sheffield were asked to participate between November 2009 and May 2010.

\section{Ethical Approval}

The design of the study was given a favourable ethical opinion by the South Yorkshire Research Ethics Committee (reference number 09/H1310/64). Patients and their partners were asked to sign three copies of a consent form - one to be kept in the patients' medical records, one for the patient or partner to keep for reference and one for the researcher.

\section{Subjects}

Three groups of pregnant women were recruited into the study along with one group of partners. Participants were excluded from the study if they had a current or past history of depression, anxiety or a significant medical condition that requires medication. Women with vaginal bleeding in the past two weeks or women who had suffered a previous miscarriage or had a family history of genetic disorder were also excluded.

The Q1 group were women recruited at booking - up to 18 weeks gestation - before they had any screening for Down's syndrome. Of 307 women approached, 107 were excluded whilst 200 women were recruited.

The Q2 group consisted of women who attended the feto-maternal unit and opted for an invasive genetic test following a screen-positive prenatal test for Down's syndrome or at the request of the pregnant woman due to maternal age. These women were between 12 and 18 weeks in gestation. Men who accompanied their spouses to this appointment were also recruited. Of 30 women and 24 partners who agreed to participate, 16 women and 4 men were excluded. Hence 14 women and 20 partners were included in the invasive test group. In total 11 couples were included. 
The Q3 'anomaly scan' group consisted of women who were screen-negative for Down's syndrome and were recruited following a routine detailed scan at 18 to 22 weeks gestation that identified no major fetal abnormalities. 101 women were recruited in this group, of which 20 were excluded. Hence 81 women were included in the anomaly scan group.

\section{Measures}

Anxiety and stress were assessed using the Beck Anxiety Inventory (BAI) [22]. The inventory consists of 21 items descriptive of subjective, somatic, or panic-related symptoms of anxiety. Self-reported answers are based on a 4point Likert scale ranging from responses of 'not at all' to 'severe' in terms of the experience of that symptom over the past month. A high total score indicates more severe levels of anxiety.

Attachment was measured using the Maternal Antenatal Attachment Scale (MAAS) [23]. The scale consists of two factors - 'quality of attachment' which represents the quality of a mother's affective experiences such as closeness and tenderness; and 'time spent in attachment mode' which represents the intensity of preoccupation a mother experiences assessed through 19 items based on the feelings, behaviours and attitudes towards the fetus. The items are rated on a 5-point Likert scale. The total attachment score is computed by combining the two subscales. High scores reflect a positive quality of attachment. The paternal version of MAAS - the Paternal Antenatal Attachment Scale (PAAS) - consists of 16 items.

\section{Survey Instruments}

Each participant was administered a survey instrument that included basic demographic information such as age, ethnicity, annual income, obstetric history, marital status and education; a scale for the assessment of anxiety and a scale for the assessment of prenatal attachment. They were asked to either complete the questionnaire while they waited or to post it back using the freepost envelope provided. Addtional file 1 gives an example of one of the questionnaires.

\section{Analyses}

Data was entered into and analysed using SPSS 16.0 (Statistical Package for Social Science, Inc., Chicago, IL) for Windows. We compared maternal attachment and anxiety scales between the three groups using descriptive statistics, analysis of variance, T-tests and MannWhitney $U$ tests as were appropriate.

We also employed multiple regression analysis, adjusting for the potential confounding effects of maternal age and parity on anxiety and attachment scores. Paternal data was described and anxiety compared with women in the invasive test group.

\section{Results \\ Demographics}

Table 1 shows the demographics of the women included in the booking $(\mathrm{Q} 1)$, invasive test $(\mathrm{Q} 2)$ and anomaly scan (Q3) groups.

Participants in Q2 were older than women in the Q1 and Q3 $(\mathrm{P}<0.001) .15$ women in Q1 $(7.5 \%), 1$ woman in Q2 (7.1\%) and 6 women in Q3 (7.4\%) were current smokers with a range of 1 - 20 cigarettes smoked a day.

\section{Anxiety}

The mean total score on the BAI was 8.34 for Q1 (SD: 7.47) ranging from $0-41 ; 12.36$ for $\mathrm{Q} 2$ (SD: 4.47) ranging from 4 - 20; and 8.19 for Q3 (SD: 7.43) ranging from 0 - 43. The invasive test group (Q2) had significantly higher levels of anxiety compared to the booking $(\mathrm{P}=0.003)$ and anomaly scan groups $(\mathrm{P}=0.002)$. This can be shown graphically in Figure 1f.

If we classify levels of anxiety, a score of 0-7 indicates minimal anxiety; 8-15 indicates mild anxiety; 16-25 indicates moderate anxiety and a score of 26 and above indicates severe anxiety [22]. Women in Q2 experienced more severe anxiety, albeit within the 'minimal' range, on average (mean: 2.29; SD: 0.79 vs. mean: 1.56; SD: 0.61 for Q1 and mean: 1.54; SD: 0.82 for Q3) but with $35.7 \%(n=5)$ experiencing moderate anxiety.

\section{Attachment}

Table 2 shows the mean total MAAS scores by group. Higher levels of attachment were experienced by women of increasing gestation (Q1 vs. Q3 P $<0.001)$. Using the cut-off scores employed by Pollock and Percy in

Table 1 clinical characteristics of women at booking, undergoing an invasive test and after an anomaly scan.

\begin{tabular}{lccc}
\hline & $\begin{array}{c}\text { Booking } \\
\text { Group } \\
\mathbf{( Q 1 )} \\
\mathbf{n = 2 0 0}\end{array}$ & $\begin{array}{c}\text { Invasive } \\
\text { Test } \\
\text { Group (Q2) } \\
\mathbf{n = 1 4}\end{array}$ & $\begin{array}{c}\text { Anomaly } \\
\text { Scan } \\
\text { Group (Q3) } \\
\mathbf{n = 8 1}\end{array}$ \\
\hline Maternal age (years) & $30.4(5.1)$ & $36.7(5.8)$ & $30.0(5.5)$ \\
Gestation (weeks) & $12[8-18]$ & $13.5[11-21]$ & $20[18-27]$ \\
Gravidity & $1[1-7]$ & $1.5[1-6)$ & $1[0-7]$ \\
Parity & $0[0-5]$ & $0[0-5]$ & $0[0-4]$ \\
Ethnicity: White & $81.5 \%$ & $78.6 \%$ & $77.8 \%$ \\
British & & & $80.2 \%$ \\
Education: Further & $76.0 \%$ & $71.4 \%$ & $66.7 \%$ \\
Marital Status: & $60.0 \%$ & $14.3 \%$ & $76.5 \%$ \\
Married & & & $71.4 \%$ \\
Employed: Yes & $78.5 \%$ & &
\end{tabular}

Data are shown as mean (SD) or median [range]. 


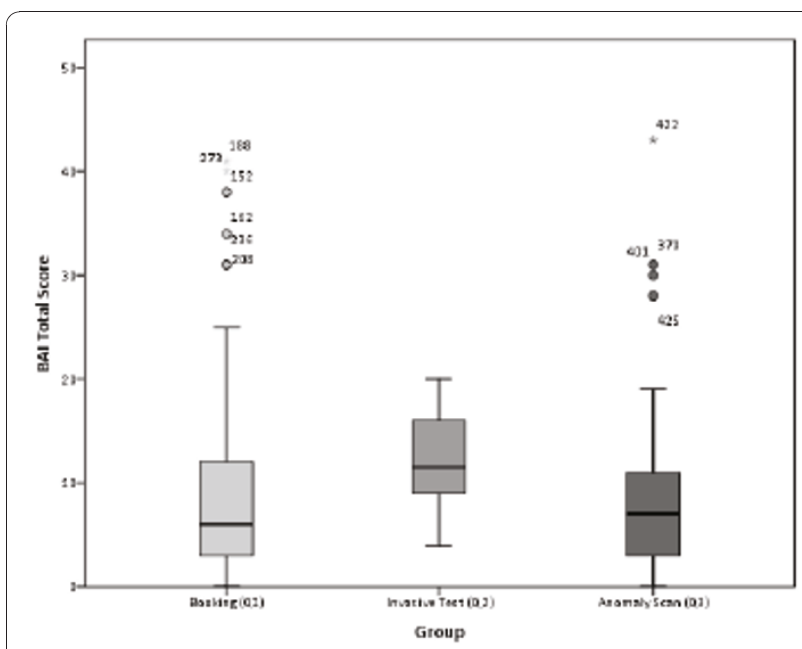

Figure 1 Distribution of total anxiety scores between groups; the mean anxiety score is higher for Q2 than the other two cohorts.

previous studies, attachment assessed by MAAS may be categorised into 'high' (a score of seventy-six and above) and 'low' [24,25]. Given these categories, the further along a woman was in her pregnancy, the 'higher' her attachment was. 49\% of women in Q1 had 'high' attachment compared to $64.2 \%$ of women in Q3 ( $\mathrm{P}=0.021)$.

Looking independently at the subscales of MAAS, $16.5 \%$ of women in Q1, $14.3 \%$ of women in Q2 and $33.3 \%$ of women in Q3 had a 'positive' quality of attachment (scoring 49 or above) suggesting that quality of attachment increases throughout pregnancy (Q1 vs. Q3 $\mathrm{P}=0.002$ )

In terms of intensity of preoccupation with their babies, 42.5\% of women in Q1, 57.1\% of women in Q2 and $53.1 \%$ of women in Q3 were 'preoccupied' (scoring 27 or above) rather than 'disinterested'.

Figure 2 shows that moderate positive correlation was found between anxiety and prenatal attachment in women in the invasive test group $(\mathrm{r}=0.479, \mathrm{n}=14, \mathrm{P}=$ 0.083 ) with the variables sharing $23 \%$ of their variance. No such correlation was demonstrated for women in Q1 and Q3.

Table 2 total BAI and MAAS scores by group.

\begin{tabular}{lccc}
\hline & $\begin{array}{c}\text { Booking } \\
\text { Group } \\
\text { (Q1) } \\
\mathbf{n = 2 0 0}\end{array}$ & $\begin{array}{c}\text { Invasive Test } \\
\text { Group } \\
\text { (Q2) } \\
\mathbf{n}=\mathbf{1 4}\end{array}$ & $\begin{array}{c}\text { Anomaly Scan } \\
\text { Group } \\
\text { (Q3) } \\
\mathbf{n}=\mathbf{8 1}\end{array}$ \\
\hline Total BAI score & $8.3(7.5)$ & $12.4(4.5)$ & $8.2(7.4)$ \\
Total MAAS & $74.7(7.1)$ & $75.2(7.1)$ & $78.5(7.1)$ \\
score & & & \\
\hline
\end{tabular}

Data are shown as mean (SD).

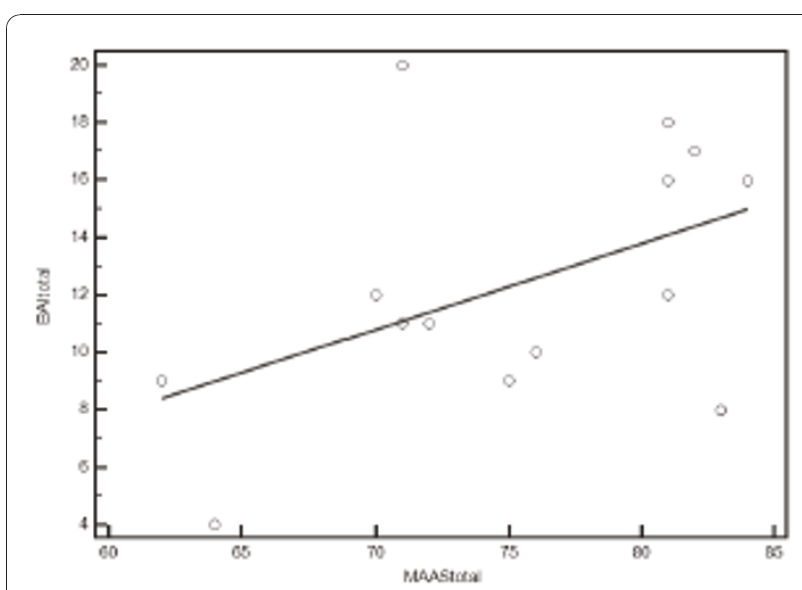

Figure 2 Correlation between total BAI and MAAS scores in $\mathrm{Q} 2$; there is moderate positive correlation between the two variables.

Table 3 shows the anxiety scores for women in each group by attachment subscales. Women who had a 'negative' quality of attachment and were more 'preoccupied' with their pregnancy had higher levels of anxiety. There was a statistically significant difference in the proportion of women in Q2 who had 'high' attachment experiencing higher levels of anxiety (mean: 13.86; SD: 3.85) compared to Q1 (mean: 9.11; SD: 7.97; P = 0.015) and Q3 (mean: 8.48; SD: 7.35; P = 0.004). Women in Q2 who had a 'negative' quality of attachment or were 'preoccupied' with their pregnancy were also more likely to experience higher levels of anxiety than any other group $(\mathrm{P}<0.05)$.

Eight women were recruited on more than one occasion (into two groups of the study) allowing for longitudinal analyses. For the five women recruited into Q1 and $\mathrm{Q} 3$, anxiety decreased over time $(\mathrm{P}=0.025)$ and attachment increased $(\mathrm{P}<0.001)$. For the three women recruited into Q1 and Q2, anxiety levels appeared to dramatically increase after undergoing an invasive test and attachment levels appeared to fall but due to the small sample size these results were not statistically significant.

\section{Paternal Data}

The mean total BAI score for partners included in Q2 was 8.20 (SD: 7.13), with scores ranging from $0-27$. This is less than women included in this group suggesting that men experience lower levels of anxiety than their partners who have recently undergone an invasive test $(\mathrm{P}=0.036)$.

The mean total PAAS score for partners included in Q2 was 54.05 (SD: 9.34), with scores ranging from 35 68. This represents mean $\%$ scores of the total maximum 
Table 3 mean total BAI scores for MAAS attachment subscales by group.

\begin{tabular}{|c|c|c|c|c|}
\hline & & \multicolumn{3}{|c|}{ Group Total BAI Scores } \\
\hline & & $\begin{array}{l}\text { Booking Group } \\
\text { (Q1) } n=200\end{array}$ & $\begin{array}{c}\text { Invasive Test } \\
\text { Group (Q2) } \\
\text { n = 14 }\end{array}$ & $\begin{array}{c}\text { Anomaly Scan } \\
\text { Group (Q3) } \\
n=81\end{array}$ \\
\hline \multirow[t]{2}{*}{ Maternal total score (global attachment) } & Low & $7.6(6.9)$ & $10.9(4.8)$ & $7.7(7.7)$ \\
\hline & High & $9.1(8.0)$ & $13.9(3.9)$ & $8.5(7.4)$ \\
\hline \multirow[t]{2}{*}{ Quality of attachment } & Negative & $9.0(7.7)$ & $11.9(4.2)$ & $8.8(6.8)$ \\
\hline & Positive & $5.0(4.9)$ & $15.0(7.1)$ & $6.9(8.6)$ \\
\hline \multirow[t]{2}{*}{ Intensity of Preoccupation } & Disinterested & $7.0(6.0)$ & $11.0(5.2)$ & $6.9(6.1)$ \\
\hline & Preoccupied & $10.2(8.8)$ & $13.4(3.9)$ & $9.4(8.3)$ \\
\hline
\end{tabular}

Data are shown as mean (SD).

scores possible of $68.1 \%(54.1 / 80)$ compared to pregnant women scores of $79.2 \%$ (75.2/95).

For the 11 couples included in Q2, pregnant women experienced considerably higher levels of anxiety than their partners (mean: 13.55; SD: 3.93 vs. mean: 6.36; SD: 8.06; $\mathrm{P}=0.015)$.

\section{Discussion}

This study shows that women who have recently undergone an invasive test have higher levels of anxiety compared to women at earlier gestations first attending for antenatal care, and to women at a later gestation in mid-trimester immediately following a reassuring fetal morphology scan. This is consistent with findings from previous studies $[5,6,26,27]$. In a study with a longitudinal design Marteau et al found that anxiety levels in this group of women later fell to lower levels compared to other women, illustrating the long-term reassurance such a procedure may offer. This observation is likely to represent a normal response to the stress of making a difficult and risky decision and may aid effective decision-making.

Maternal stress has been shown to have severe effects on fetal brain development. Prenatal stress exposure during the development of the hippocampus, the component of the brain responsible for spatial memory tasks and associative and procedural memory, has been shown to decrease formation of synapses by at least $30 \%$ [28]. Offspring exposed to prenatal stress also show decreased numbers of glucocorticoid messenger ribonucleic acid (mRNA) and increased levels of corticotropinreleasing hormone $(\mathrm{CRH})$ expression in the amygdala $[7,8]$. These changes may lead to a predisposition to mental health disorders such as depression as well as an increased sensitivity to stressful situation with a prolonged recovery and difficulties regulating emotions [9]. Indeed Van der Bergh found that prenatal anxiety in the third trimester of pregnancy was positively correlated with a difficult temperament in infants at 10 weeks and 7 months of age [29].
Prenatal stress has also been shown to affect behavioural development, thymic function and immune function in animal studies. Rhesus monkeys that were prenatally stressed have been shown to have lower amounts of exploratory behaviour and significantly more disturbance behaviour compared to controls [30]. Rats subjected to synthetic glucocorticoids prenatally showed a decrease in number of thymic $\mathrm{T}$ cells postnatal [31].

The effect of stress and its' associated immune changes in pregnancy may be a major factor in preterm labour. Two hypotheses have been put forward - firstly, that preterm birth may occur due to the significant immunological changes that occur as a result of stress without subsequent infection [32] and secondly, that preterm birth occurs through increased susceptibility to infections [33].

Stress has also been shown to decrease levels of the pregnancy supporting hormone progesterone and progesterone induces blocking factor (PIBF) which up regulates the production of Th1 cytokines [13,34]. This leads to increased prostaglandin production and increased uterine contractility and may cause pre-term labour [34].

Infection and inflammation account for up to $30 \%$ of preterm deliveries [35] and bacterial vaginosis, the most commonly associated infection with pre-term delivery, is more prevalent in pregnant women undergoing greater psychological stress [36].

These findings highlight the additional complications a woman and her unborn child may be at risk of as a result of the increased levels of anxiety associated with undergoing an invasive genetic test in pregnancy our observations have shown.

There is an increase in the total fetal attachment score from a pregnant women's booking visit to her fetal morphology scan in mid-trimester which many studies have also reported $[24,37,38]$. At variance with our observations, Lawson et al reported that women who opted for an invasive test had higher levels of attachment than women at screening or who had no test [19]. However 
our invasive test group demonstrated moderate positive correlation between anxiety and attachment, suggesting that heightened anxiety at the time of an invasive test may be a cause or consequence of stronger feto-maternal attachment. This is at variance with some previous studies that suggest that anxiety leads to a decrease in the quality of attachment, and that a lack of attachment may lead to increased anxiety [24,39-41]. Our observation supports the hypothesis that women who develop an emotional attachment to the fetus are more likely to experience anxiety about the pregnancy and unborn child $[42,43]$ and that women who experience low levels of attachment are likely to feel less anxious about their pregnancy.

Partners have been shown to experience less anxiety than their respective partners, supporting previous research [20,21]. The difference in anxiety between women and their partners may be explained by the simple actuality that it is the pregnant woman who is carrying the baby and undergoes any invasive procedure rather than her partner and is therefore likely to have additional anxiety related to the pain and possible complications of that procedure.

\section{Study Limitations and Future Work}

Considering the potential long-term benefits of undergoing an invasive test in terms of the reduction in anxiety levels it would be worthwhile undertaking longitudinal studies of women who have undergone invasive testing throughout pregnancy and into the postnatal period.

Most previous studies used the Spielberger State-Trait Anxiety Inventory (STAI) or the Pregnancy-Related Anxiety Questionnaire (PRAQ-r) to assess anxiety. The BAI is a widely employed survey instrument that focuses most on somatic symptoms in order to avoid correlation with depression as well as anxiety [44]. A study utilising both the BAI and STAI in adult psychiatric outpatients found that both scales demonstrated high internal reliabilities but that the BAI proved more useful as a screening instrument for a current anxiety disorder than the STAI $[45,46]$. However the BAI gives no indication of a woman's underlying 'trait' anxiety. Kowalcek et al found that women who had an increased trait anxiety score were more likely to have an increased state anxiety score after an invasive test [47]. The recently developed Beck Anxiety Inventory-Trait (BAIT) may be a useful survey instrument for future research [48]. Although we have not determined trait anxiety in our cohort of pregnant women we have tried to minimise the confounding influence of this on our observation by excluding women with previous histories of anxiety and depression.

Our pilot data on paternal anxiety and attachment in relation to invasive prenatal diagnosis should inform larger sufficiently powered studies aimed at elucidating the complex factors that are likely to influence feto-paternal attachment. Furthermore future work exploring the potential impact of clinical care interventions on maternal anxiety and fetal attachment are needed to optimise care for this client group.

\section{Conclusion}

Women undergoing prenatal diagnostic procedures will experience more psychological distress and anxiety compared to women who are not at an increased risk of carrying a fetus affected by a chromosomal abnormality. Increased anxiety levels may in turn impact upon the extent to which a women and her partner can start to form a bond with their unborn child. It is therefore important to identify anxiety in pregnancy in order to offer each woman and her partner the most favourable environment and opportunities for a healthy pregnancy and relationship with their child.

The establishment of a multidisciplinary treatment situation, in which access to psychological support is offered, may be extremely beneficial for these women who are at an increased risk of experiencing anxiety.

\section{Author's Information}

SJA conducted this research as part of her Bachelor of Medical Sciences Honours degree under the supervision of DOCA and with the help of JS.

\section{Additional material}

Additional File 1: Questionnaire for Booking Group (Q1). A

questionnaire for women recruited at booking following a dating scan. The questionnaire includes sections on demographics, a scale to assess anxiety (BAI) and a scale to assess attachment (MAAS).

Acknowledgements and Funding

There were no sources of funding. SJA carried out all parts of the work presented with the exception of the aforementioned

\section{Author details}

${ }^{1}$ Academic Unit of Reproductive and Developmental Medicine, University of Sheffield, 4th Floor, Jessop Wing, Tree Root Walk, Sheffield, S10 2SF, UK. ${ }^{2}$ Academic Unit of Reproductive and Developmental Medicine, University of Sheffield, South Yorkshire, UK. ${ }^{3}$ Academic Unit of Reproductive and Developmental Medicine, University of Sheffield, South Yorkshire, UK.

\section{Authors' contributions}

SJA participated in the study design, recruited patients into the study, performed the statistical analysis and drafted the manuscript. JS helped to recruit patients into the study. DOCA conceived the study, participated in its design and coordination and performed some of the statistical analysis. All authors read and approved the final manuscript.

\section{Competing interests}

The authors declare that they have no competing interests.

Received: 24 January 2011 Accepted: 12 July 2011

Published: 12 July 2011 


\section{References}

1. Austin MP, Leader L: Maternal stress and obstetric and infant outcomes: epidemiological findings and neuroendocrine mechanisms. Australian \& New Zealand Journal of Obstetrics \& Gynaecology 2000, 40(3):331-7.

2. Kowalcek I, Huber G, Lammers C, Brunk J, Bieniakiewicz I, Gembruch U: Anxiety scores before and after prenatal testing for congenital anomalies. Archives of Gynecology \& Obstetrics 2003, 267(3):126-9.

3. Marteau TM, Johnston M, Shaw RW, Michie S, Kidd J, New M: The impact of prenatal screening and diagnostic testing upon the cognitions, emotions and behaviour of pregnant women. Journal of Psychosomatic Research 1989, 33(1):7-16.

4. Kleinveld $\mathrm{JH}$, Timmermans DR, de Smit DJ, Ader HJ, van der Wal G, ten Kate LP: Does prenatal screening influence anxiety levels of pregnant women? A longitudinal randomised controlled trial. Prenatal Diagnosis 2006, 26(4):354-61

5. CC Ng, Lai FM, Yeo GS: Assessment of maternal anxiety levels before and after amniocentesis.[see comment]. Singapore Medical Journal 2004, 45(8):370-4.

6. Sarkar P, Bergman K, O'Connor TG, Glover V: Maternal antenatal anxiety and amniotic fluid cortisol and testosterone: possible implications for foetal programming. Journal of Neuroendocrinology 2008 20(4):489-96.

7. Bale TL: Is mom too sensitive? Impact of maternal stress during gestation. Frontiers in Neuroendocrinology 2005, 26(1):41-9.

8. Buitelaar JK, Huizink AC, Mulder EJ, Robles de Medina PG, Visser GHA Prenatal stress and cognitive development and temperament in infants. Neurobiology of Aging 2003, 24:553-S60.

9. Ruiz RJ, Avant KC: Effects of maternal prenatal stress on infant outcomes: a synthesis of the literature. Advances in Nursing Science 2005, 28(4):345-55.

10. Welberg L, Seckl J, Holmes M: Inhibition of 11 beta-hydroxysteroid dehydrogenase, the foeto-placental barrier to maternal glucocorticoids, permanently programs amygdala GR mRNA expression and anxiety-like behaviour in the offspring. European Journal of Neuroscience 2000, 12:1047-1054.

11. Field T, Diego M, Hernandez-Reif M: Prenatal depression effects on the fetus and newborn: a review. Infant Behaviour and Development 2006, 29:445-455.

12. Hales CN, Barker DJ: The thrifty phenotype hypothesis. British Medical Bulletin 2001, 60:5-20.

13. Knackstedt MK, Hamelmann E, Arck PC: Mothers in stress: consequences for the offspring. American Journal of Reproductive Immunology 2005 54(2):63-9.

14. Uno H, Eisele S, Sakai A, Shelton S, Baker E, DeJesus O: Neurotoxicity of glucocorticoids in the primate brain. Hormones and Behaviour 1994 28:336-348.

15. Diego MA, Jones NA, Field T, Hernandez-Reif M, Schanberg S, Kuhn C: Maternal psychological distress, prenatal cortisol, and fetal weight. Psychosomatic Medicine 2006, 68:747-753.

16. Muller ME: Development of a prenatal attachment inventory. Western Journal of Nursing Research 1993, 15:199-215.

17. Van den Berg B, Simons A: A review of scales to measure the motherfoetus relationship. Journal of Reproductive and Infant Psychology 2009, 27(2):114-126.

18. Kleinveld JH, Timmermans DR, van der Berg M, van Eijk JT, ten Kate LP: Does offering and performing prenatal screening influence women's attachment to their unborn child? A longitudinal randomized controlled trial. Prenatal Diagnosis 2007, 27(8):757-64.

19. Lawson KL, Turriff-Jonasson SI: Maternal serum screening and psychological attachment to pregnancy. Journal of Psychosomatic Research 2006, 60(4):371-8

20. Kowalcek I, Muhlhoff S, Bachmann S, Gembruch U: Depressive reactions and stress related to prenatal medicine procedures. Ultrasound in Obstetrics and Gynaecology 2002, 19:18-23.

21. Sahin NH, Gungor I: Congenital anomalies: parents' anxiety and women's concerns before prenatal testing and women's opinions towards the risk factors. Journal of Clinical Nursing 2008, 17(6):827-36.

22. Beck AT, Steer RA: Manual for the Beck Anxiety Inventory. San Antonio, Texas: Psychological Corporation; 1990.
23. Condon JT: The assessment of antenatal emotional attachment Development of a questionnaire instrument. British Journal of Medical Psychology 1993, 66:167-183.

24. Hart R, McMahon CA: Mood state and psychological adjustment to pregnancy. Archives of Women's Mental Health 2006, 9:329-337.

25. Pollock PH, Percy A: Maternal antenatal attachment style and potential fetal abuse. Child Abuse and Neglect 1999, 23(12):1345-1357.

26. Marteau TM, Kidd J, Cook R, Michie S, Johnston M, Slack J, Shaw RW: Psychological effects of having amniocentesis: are there due to the procedure, the risk or the behaviour? Journal of Psychosomatic Research 1992, 36(4):395-402.

27. Sarkar P, Bergman K, Fisk NM, Glover V: Maternal anxiety at amniocentesis and plasma cortisol. Prenatal Diagnosis 2006, 26(6):505-9.

28. Hayashi A, Nagaoka M, Yamada K, Ichitani Y, Miake Y, Okado N: Maternal stress induces synaptic loss and developmental disabilities of offspring. International Journal of Developmental Neuroscience 1998, 16:209-216.

29. Van den Bergh B: Maternal emotions during pregnancy and fetal and neonatal behavior. In Fetal Behaviour: Developmental and Perinatal Aspects. Edited by: Nijhuis JG. Oxford University Press: Oxford; 1992:157-178.

30. Schneider ML: The effect of mild stress during pregnancy on birth weight and neuromotor maturation in rhesus monkey infants (Macaca mulatta). Infant Behaviour and Development 1992, 15:389-403.

31. Bakker JM, Schmidt ED, Kroes H: Effects of short-term dexamethasone treatment during pregnancy on the development of the immune system and the hypothalamo-pituitary adrenal axis in the rat. Journal of Neuroimmunology 1995, 63(2):183-191.

32. Ruiz RJ, Pearson AJ: Psychoneuroimmunology and preterm birth. A holistic model for obstetrical nursing, practice and research. Maternal Child Nursing 1999, 24:230-235.

33. Wadhwa PD, Culhane JF, Rauh V, Barve SS, Hogan V, Sandman CA, Hobel CJ, Chicz-DeMet A, Dunkel-Schetter C, Garite TJ, Glynn L: Stress, infection and preterm birth: a biobehavioural perspective. Paediatric and Perinatal Epidemiology 2001, 15(Suppl 2):17-29.

34. Gennaro S, Hennessy MD: Psychological and physiological stress: impact on preterm birth. JOGNN - Journal of Obstetric, Gynecologic, \& Neonatal Nursing 2003, 32(5):668-75

35. Rich-Edwards JW, Grizzard TA: Psychosocial stress and neuroendocrine mechanisms in preterm delivery. American Journal of Obstetrics \& Gynecology 2005, 192(5 Suppl):S30-5.

36. Culhane JF: Exposure to chronic stress on ethnic differences in rates of bacterial vaginosis amongst pregnant women. American Journal of Obstetrics \& Gynecology 2002, 187:1272-1276.

37. Hjelmstedt A, Widstrom AM, Collins A: Psychological correlates of prenata attachment in women who conceived after in vitro fertilisation and women who conceived naturally. Birth 2006, 33(4):303-310.

38. Zachariah R: Attachment, social support, life stress, and psychological well-being in pregnant low-income women: A pilot study. Clinical Excellence for Nurse Practioners 2004, 8(2):60-67.

39. Anderson VN, Fleming AS, Steiner M: Mood and the transition to motherhood. Journal of Reproductive and Infant Psychology 1994, 12:69-77.

40. Armstrong D, Hutti M: Pregnancy after perinatal loss: the relationship between anxiety and prenatal attachment. Journal of Obstetric, Gynecologic \& Neonatal Nursing 1998, 27(2):183-189.

41. Condon JT, Corkindale J: The correlates of antenatal attachment in pregnant women. British Journal of Medical Psychology 1997, 70:359-372

42. Leifner M: Psychological effects of motherhood: A study of first pregnancy. New York: Praeger; 1980.

43. Shereshefsky PM, Yarrow LJ: Psychological aspects of a first pregnancy and early postnatal adaptation. New York: Raven; 1973.

44. Bieling J, Antony MM, Swinson RP: The State-Trait Anxiety Inventory: Structure and content re-examined. Behavioural Research and Therapy 1998, 36:777-788.

45. Kabacoff Rl, Segal DL, Hersen M, Van Hasselt VB: Psychometric properties and diagnostic utility of the Beck Anxiety Inventory and the state-trait anxiety inventory with older adult psychiatric outpatients. Journal of Anxiety Disorders 1997, 11(1):33-47.

46. Osman A, Kopper BA, Barrios FX, Osman JR, Wade T: The Beck Anxiety Inventory: Re-examination of Factor Structure and Psychometric Properties. Journal of Clinical Psychology 1997, 53(1):7-14 
47. Kowalcek I, Gembruch U: Pregnant women's cognitive concept concerning their unborn prior to prenatal diagnosis. Fetal Diagnosis \& Therapy 2008, 24(1):22-8.

48. Kohn PM, Kantor L, DeCicco TL, Beck AT: The Beck Anxiety Inventory-Trait (BAIT): a measure of dispositional anxiety not contaminated by dispositional depression. Journal of Personality Assessment 2008, 90(5):499-506.

Pre-publication history

The pre-publication history for this paper can be accessed here: http://www.biomedcentral.com/1472-6874/11/33/prepub

doi:10.1186/1472-6874-11-33

Cite this article as: Allison et al.: The effect of stress and anxiety associated with maternal prenatal diagnosis on feto-maternal attachment. BMC Women's Health 2011 11:33.

Submit your next manuscript to BioMed Central and take full advantage of:

- Convenient online submission

- Thorough peer review

- No space constraints or color figure charges

- Immediate publication on acceptance

- Inclusion in PubMed, CAS, Scopus and Google Scholar

- Research which is freely available for redistribution

Submit your manuscript at www.biomedcentral.com/submit 